NISTIR 7721

\title{
Recognizing People from Dynamic and Static Faces and Bodies: Dissecting Identity with a Fusion Approach
}

O’Toole, A. J. Phillips, P. J.

Weimer, S. Roark, D. A. Ayadd, J. Barwick, R. Dunlop, J. 
NISTIR 7721

\section{Recognizing People from Dynamic and Static Faces and Bodies: Dissecting Identity with a Fusion Approach}

O’Toole, A. J. The University of Texas at Dallas

Phillips, P. J. NIST

Information Access Division

Weimer, S. Roark, D. A. Ayadd, J.

Barwick, R. Dunlop, J. The University of Texas at Dallas

September 2010

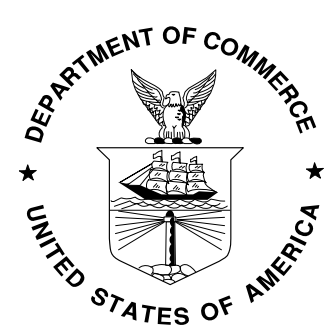

U.S. Department of Commerce Gary Locke, Secretary 


\title{
Recognizing people from dynamic and static faces and bodies: Dissecting identity with a fusion approach
}

\author{
Alice J. O'Toole ${ }^{\mathrm{a}}$, P. Jonathon Phillips ${ }^{\mathrm{b}}$, Samuel Weimer ${ }^{\mathrm{a}}$, Dana A. Roark ${ }^{\mathrm{a}}$, \\ Julianne Ayyad ${ }^{\mathrm{a}}$, Robert Barwick ${ }^{\mathrm{a}}$, Joseph Dunlop ${ }^{\mathrm{a}}$ \\ ${ }^{a}$ The University of Texas at Dallas \\ ${ }^{b}$ National Institute of Standards and Technology
}

\begin{abstract}
The goal of this study was to evaluate human accuracy at identifying people from static and dynamic presentations of faces and bodies. Participants matched identity in pairs of videos depicting people in motion (walking or conversing) and in "best" static images extracted from the videos. The type of information presented to observers was varied to include the face and body, the face only, and the body only. Identification performance was best when people viewed the face and body in motion. There was an advantage for dynamic over static stimuli, but only for conditions that included the body. Control experiments with multiple-static images indicated that some of the motion advantages we obtained were due to seeing multiple images of the person, rather than to the motion, per se. To computationally assess the contribution of different types of information for identification, we fused the identity judgments from observers in different conditions using a statistical learning algorithm trained to optimize identification accuracy. This fusion achieved perfect performance. The condition weights that resulted suggest that static displays encourage reliance on the face for recognition, whereas dynamic displays seem to direct attention more equitably across the body and face.
\end{abstract}

Keywords: face, dynamic, gait

\section{Introduction}

In the real world, we interact with people in motion. These interactions typically begin at a distance and unfold over time, as a person approaches, and ultimately stands "face-to-face" with us. The recognition of a person in 
natural viewing conditions, therefore, begins with a glimpse at the overall shape of a person and builds toward more confident judgments as the particularities of the movements, body structure, and face are integrated and processed.

The human face has generally been regarded as the most easily accessible and accurate entry point into the task of determining a person's identity from visual cues. Despite evidence that humans excel at recognizing familiar faces (Burton, Bruce, and Hancock, 1999a), performance is less impressive for relatively unfamiliar faces (Hancock, Bruce, and Burton, 2000). In particular, there is evidence to indicate that recognition can be poor under viewing conditions that are non-optimal or are poorly matched to those in which a face is learned. The difficulties humans have with unfamiliar face recognition can be mitigated potentially by relying on a broader array of identity cues available in natural viewing conditions. These include the shape and structure of the body, as well as gait and other gesture-based movements of the body. Body motions and gestures that are idiosyncratic or "identitydiagnostic" have been referred to previously as dynamic identity signatures (O'Toole, Roark, and Abdi, 2002).

There is surprisingly little psychological data on the extent to which humans use information, beyond the face, to identify people. To the best of our knowledge, there is only one demonstration of person recognition from a pure body motion cue (Cutting and Kozlowski, 1977). In that study, Cutting and Kozlowski (1977) found poor, but above chance performance, for identifying friends from point-light motion displays. Using more natural dynamic viewing conditions, Burton, Wilson, Cowan, and Bruce (1999b) considered the relative contribution of the face versus body for recognition in dynamic viewing conditions. They looked at identification of people captured on CCTV as they walked through a door and found that observers performed quite poorly when they were unfamiliar with the person in the video, but were nearly perfect when the person was known to them. In a related study, Davis and Valentine (2008) confirmed the finding that matching unfamiliar identities in video is highly susceptible to error. They found this across a range of conditions that included low-, moderate- and high-quality video. Burton, Wilson, Cowan, and Bruce (1999b) also found that identification performance declined substantially when the face in the video was obscured, but remained high when the body was obscured. This result suggests that even with more complete information about the face and body, recognition performance is supported more strongly by the face than by the other information in the 
video.

In static displays, Robbins and Coltheart (2010) likewise demonstrated the importance of the face in identifying relatively unfamiliar people. In that study, observers learned people from full body pictures and were tested with composite images made from the head of one person on the body of another person. People were more accurate at identifying people from their heads than from their bodies. Moreover, in integrating information from the combined face and body, Robbins and Coltheart (2010) found a greater degree of holistic processing across the right-left mid-line halves than across the top- and bottom- halves of the full body image. They conclude that the head is more important than the body for recognition, but that the body can also provide identity information, when the person is processed as an integrated whole.

In the context of viewing people in motion, Pilz, Vuong, Bülthoff, and Thornton (2006) have also considered the question of how we integrate information across the face and body in making an identification decision. They placed three-dimensional head models from different people onto a single identical moving body, defined by an avatar. Observers responded more quickly to a target face when the body was approaching than when it was receding. In a second experiment, they found that faces learned on an approaching avatar, were responded to more quickly than those learned on an avatar that receded. These findings suggest that natural approach motions may facilitate the processing of a face. However, the body information in the Pilz et al. (2006) study did not vary. Thus, it remains an open question if approach motion would likewise facilitate the processing of the body if it carried individuating information.

From a neural perspective, the visual processing of faces and bodies from dynamic and static displays is likely to involve a complex network of brain regions. Based on evidence from human neuropsychology and primate neurophysiology, Haxby, Hoffman, and Gobbini (2000) proposed a distributed neural network that divides the processing of the invariant and changeable aspects of faces into two streams. According to this model, the invariant features of faces, those useful for face identification, are processed in the ventral temporal areas of the cortex near the fusiform gyrus (cf. fusiform face area, FFA, Kanwisher, McDermott, and Chun 1997). The changeable aspects of faces (e.g., expression, gaze), useful for social communication, are thought to be processed in the posterior Superior Temporal Sulcus (pSTS) along the dorsal stream of visual processing. 
As noted by Haxby et al. (2000), the invariant information in faces supports the function of identifying people, whereas the motion-based changeable information supports a social communication function. Given that the neural systems responsible for these functions are, to a first approximation, functionally and anatomically distinct, the question arises as to how facial motions contribute to face recognition. The task of recognizing someone is based presumably more on the invariant structure of a face. In theoretical terms, O'Toole et al. (2002) proposed two ways that motion could benefit face recognition. The supplemental information hypothesis posits that we represent dynamic identity signatures in addition to the invariant features of faces. The representation enhancement hypothesis posits that motion benefits face recognition by perceptual structure-from-motion processes that enable a better three dimensional representation of a face (O'Toole et al., 2002). To date, there is strong support for the supplemental information hypothesis, and hence the use of dynamic identity signatures for face recognition, but only limited support for the representation enhancement hypothesis (O'Toole and Roark, 2010, in press).

Although the Haxby et al. (2000) and O'Toole et al. (2002) models were proposed to account for face processing, some essential elements of these perspectives may apply analogously to the recognition of people from natural viewing of full bodies. It has been known for sometime that the pSTS plays an important role also in processing body motion as well as the motion of individual body parts (e.g., hands) (cf. Allison, Puce, and McCarthy 2000; Pinsk, DeSimone, Moore, Gross, and Kastner 2005). As noted, for the face, and possibly body, the role of pSTS may be primarily for processing social communication movements (Haxby et al., 2000). By extension, the pSTS may also have a role in recognition via dynamic identity signature processing (O'Toole et al., 2002). The extra-striate body area (EBA) may likewise contribute to the recognition of people from static images of bodies and body parts (Downing, Jiang, Shuman, and Kanwisher, 2001). This region, located in the lateral occipital cortex, responds to still images of bodies and body parts more strongly than it responds to a variety of control images, including faces. Downing et al. (2001) have suggested a role for the EBA in representing the visual appearance of bodies. In particular, they suggest a role for EBA in identification when viewing conditions are poor and the face is not easily accessible due to poor lighting, occlusion, or viewing direction. Some studies have also proposed a role for EBA in processing body motions with the goal of understanding actions and intent (Astafiev, Stanely, Shuman, 
and Corbetta, 2004), but this finding remains controversial (Downing, Peelen, Wiggett, and Tew, 2006; Peelen and Downing, 2005).

Combined, the data from functional neuroimaging studies indicate a widely distributed network of neural regions involved in processing faces and bodies, both from static and dynamic stimuli. These studies also suggest that neural regions may differ in the extent to which they subserve different tasks, including the processing of social signals (pSTS), the recognition of intent (pSTS, EBA), and person recognition (FFA, EBA, and pSTS). The complexity of the neural processing belies a simpler question about how humans use the information in faces and bodies for identifying someone under natural viewing conditions, when a face is attached to a body and is experienced intermittently in motion and at rest. A better understanding of how humans identify people from static and dynamic information in the face and body can constrain the interpretation of the neural data.

The goal of the present study was to systematically assess the contribution of the face and body for making an identity judgment in static versus dynamic presentation conditions. We also tested the extent to which identification advantages in video could be accounted for by the presentation of "more information about a person" from the multiple static images that comprise the video sequence. We carried out a series of experiments in which participants matched "person identity" (same or different?) in pairs of static images/videos. For all experiments, the task was to determine whether two images/videos, which appeared side by side on a computer screen, were of the same person or of different people. The experiments differed only in the type of stimulus used for the identity match. In Experiments 1a, 2a, and 3a, participants viewed pairs of videos. In Experiments 1b, 2b, and 3b, identifications were made on the "best" image extracted from the videos. The stimuli used in Experiments 1a and 1b included both the face and body. For Experiments $2 \mathrm{a}$ and $2 \mathrm{~b}$, only the face was visible and for Experiments $3 \mathrm{a}$ and $3 \mathrm{~b}$, only the body was visible. As we shall see, the face and body and body-only experiments yielded a video advantage. Therefore, we carried out multi-static control experiments (Experiments 1c and 3c) to test the extent to which the video advantage could be accounted for by the extra image-based information in the video. Table 1 gives a summary of stimulus conditions in each experiment.

Within each experiment, we also varied the types of videos presented for identity matching. In one condition, participants saw pairs of "gait" videos, picturing a person walking toward a camera. In a second condition, they saw 
pairs of "conversation" videos, picturing the subject conversing with another person. In a third condition, participants had to match the identity of the two people between a conversation and gait video. We expected performance to be best for the gait stimuli, because the quality and resolution of the final frames of these videos was best. The primary reason we used different types of match conditions was to diversify the stimulus types, allowing for a more general test of the main questions of the study. These general questions focused on video versus static presentations and recognition from the face versus body.

Next, we applied a fusion strategy to the task of quantitatively and qualitatively assessing how to optimally combine human identity judgments based on different information (face and/or body, viewed in static or dynamic displays) to improve identification. Fusion has been used widely in computer vision applications to improve biometric identifications by combining information from multiple sources (e.g., face and fingerprint, or face and iris) (Ross, Nandakumar, and Jain, 2004). In general, the idea is that when partially independent information about a person's identity is available from multiple sources, the information can be combined to improve accuracy over that of the best performing source. Fusion algorithms vary in complexity from simple averaging of the judgments from different sources to pattern classification algorithms that learn a statistical mapping from the source judgments to the identification status (e.g., same or different person). Here we used a pattern classifier based on partial least squares (PLS) regression to implement the fusion. PLS combines elements of principal components analysis (PCA) and multiple regression and provides a set of weights for the optimal combination of information across sources. These weights can be used to assess the role of different information sources in creating an optimal identity judgment. As such, they can provide insight into the extent to which the information used by humans across these presentation modes is complementary, redundant, or independent.

\section{Experimental Methods}

The methods were similar for all experiments, and so for brevity, we describe them once and include a brief section that details the stimulus manipulations undertaken in each experiment. We conducted these experiments independently using different observers so that we could use the same set of identity pairings in each experiment. This allows for the fusion across exper- 
iments to be based on independent participant judgments for single viewings of each identity pair.

\subsection{Participants}

Volunteers for the experiments were recruited from the undergraduate student population enrolled at The University of Texas at Dallas (UTD). Students received research credit as part of a course requirement for psychology majors. A minimum of 30 volunteers participated in each experiment. Exact numbers of participants for each experiment are indicated in Table 1.

\subsection{Stimuli}

A database of video clips and static images of faces and people (OToole, Harms, Snow, Hurst, Pappas, Ayyad, and Abdi, 2005) served as the source of stimuli for these experiments. There were multiple gait and conversation videos available for each person in the database. A gait video showed a person walking parallel to the line of sight of a stationary camera, starting at a distance of $10 \mathrm{~m}$. The person is filmed as they walk toward the camera and veer off to the left to pass the camera (see See Figure 1 for a multi-frame example of these videos). The gait videos varied across individuals from $8 \mathrm{~s}$ to $11 \mathrm{~s}$, depending on how quickly the individual walked. The average duration of the videos was $9.6 \mathrm{~s}$. We decided not to edit these videos to a common duration in order to preserve natural differences in walking speed and style for individuals. A conversation video showed a person conversing with a laboratory staff member. The lab member stands with his/her back to the camera and the subject faces the lab member. The distance between the camera and the center point of the subject's trajectory was 10.4 meters. The videos were filmed from the top of a short flight of stairs at a height of 3.5 meters, looking down on the subject and the lab member. To encourage gesturing in the videos, the subject was asked to give directions to a building on campus. For the experiments, these $10 \mathrm{~s}$ videos were edited to be $9.6 \mathrm{~s}$ in length to match the average of the gait videos. Both types of videos were filmed in a building foyer with high ceilings, enclosed entirely on one side with glass windows. This environment approximates outdoor lighting and makes for variable lighting conditions across the set of videos because the position and intensity of the light (mostly the sun) varies on a stimulus-by-stimulus basis. There were two sets of images and videos for each person: an original set and a second, duplicate set of images and videos collected between one week and six months subsequent to the original set. Thus, across the two 

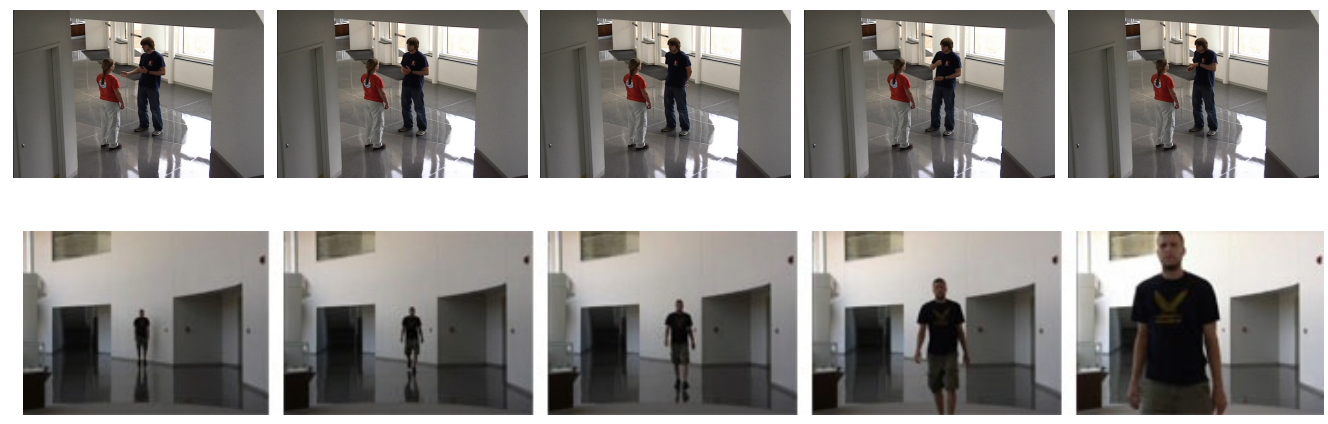

Figure 1: Five frames extracted from an example of the conversation videos (top) and the gait videos (bottom).

filming sessions, there are natural variations in the person's appearance including hairstyle, clothing, etc. This ensured that participants in the identity matching experiments could not base their decisions on transient cues such as clothing, or other artifacts.

To create stimuli for the body only experiments, we obscured the face by blurring a circular region around and including the face in each frame of the video. To create stimuli for the face only experiments, we applied a blackout mask to the entire image in each frame, exclusive of a circular bubble around the face. For the static presentations, we extracted the "best" still image from each video as follows. For the gait videos, this was the image taken closest to the camera that showed the face from the frontal view. For the conversation video, we chose a good image that showed the face from as close to a frontal view as possible. See Figure 2 for examples of the stimuli. To make stimuli for the multi-static control experiments, we extracted one image per second for each of the videos. Specifically, we took the first frame of each second and presented these frames in sequence at a rate of one image per second. The images were far enough apart in the video to eliminate apparent motion.

In all, there were 60 unique identities represented in the videos. All were young adult males between 19-30 years of age. Twenty identities were used to create identity-match pairs (i.e., two videos of the same person-presented in match trials). The remaining 40 identities were used to create no-match pairs (i.e., two videos of different people). 

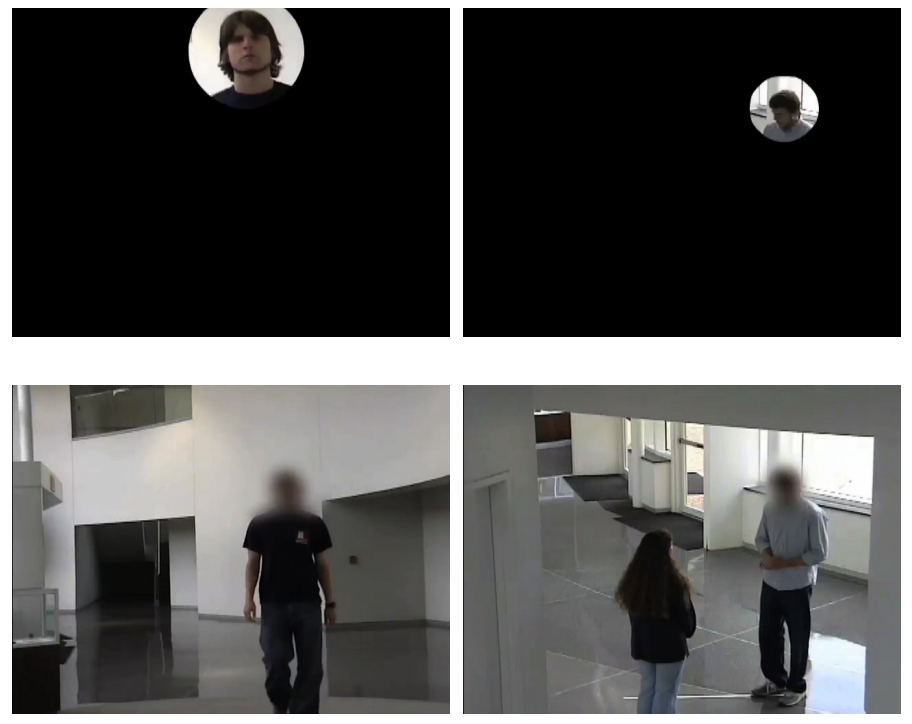

Figure 2: Five frames extracted from an example of the conversation videos (top) and the gait videos (bottom).

\subsection{Procedure}

Participants in each experiment were assigned randomly to one of three conditions. In the gait-gait (GG) condition, they matched identity in a pair of stimuli created from the gait videos (with the exact stimulus type determined by the experiment). In the conversation-conversation (CC) condition, participants matched identity in a pair of stimuli created from the conversation videos. In the conversation-gait ( $\mathrm{CG}$ ) condition, participants matched identity between stimuli created from a conversation video and a gait video.

The participants viewed pairs of videos (images) and were asked to determine if the people pictured were the "same person" or "different people". On each trial, they viewed the first video in the pair on the left side of the screen, followed by the second video presented on the right side of the screen. The screen went blank at the end of each video. For the best-static image experiments, the first image appeared on the left side of the screen for 9.6 $\mathrm{s}$ (the average duration of the videos) and the second image appeared for $9.6 \mathrm{~s}$ on the rights side of the screen. Again, the screen went blank at the end of each image presentation. Next a prompt appeared with the following response choices : "1.) sure they are the same person; 2.) think they are the same person; 3.) don't know; 4.) think they are not the same person; 5.) 
Table 1: Summary of experiments.

\begin{tabular}{llrrr}
\hline Experiment & Information & Presentation & $N$ & main effect \\
\hline 1a & face and body & video & 48 & $F(2,45)=9.21, p<.001$ \\
1b & face and body & static & 30 & $F(2,27)=1.25, p<n s$. \\
1c & face and body & multi-static & 30 & $F(2,27)=3.37, p<.05$ \\
2a & face only & video & 30 & $F(2,27)=4.54, p<.001$ \\
2b & face only & static & 36 & $F(2,33)=12.12, p<.001$ \\
3a & body only & video & 30 & $F(2,27)=10.03, p<.001$ \\
3b & body only & static & 31 & $F(2,28)=9.39, p<.001$ \\
3c & body only & multi-static & 30 & $F(2,27)=.36, p<n s$. \\
\hline
\end{tabular}

sure they are not the same person." The prompt remained visible until the participant pressed a response key.

There were 40 trials in all: 20 matched identity trials and 20 non-matched identity trials. The order of stimulus presentation was randomized for each participant.

\subsection{Results}

The confidence ratings for the identity match task enabled the construction of ROC curves. These appear in Figure 3 and offer an overview of performance across stimulus conditions (statistical tests follow). Identification performance appears best when both the face and the body were presented in motion. In more detail, these curves suggest three kinds of results. First, comparing the right-left ROC curve pairs suggests an advantage for the video over static presentations for the face and body and body-only conditions, but not for the face-only condition. Second, performance for the face and body appears to be better than performance for either the face or body alone. Moreover, identification with the face-only is far better than identification with the body-only. Third, the relative placement of the ROC curves within each experiment indicates better performance for the GG condition over the $\mathrm{CC}$ and $\mathrm{CG}$ conditions, in all but the face-only video match condition. The $\mathrm{CC}$ and $\mathrm{CG}$ conditions were roughly equivalent in all but the static face-only condition.

To examine these differences statistically, we computed a $d$ ' for discrimi- 

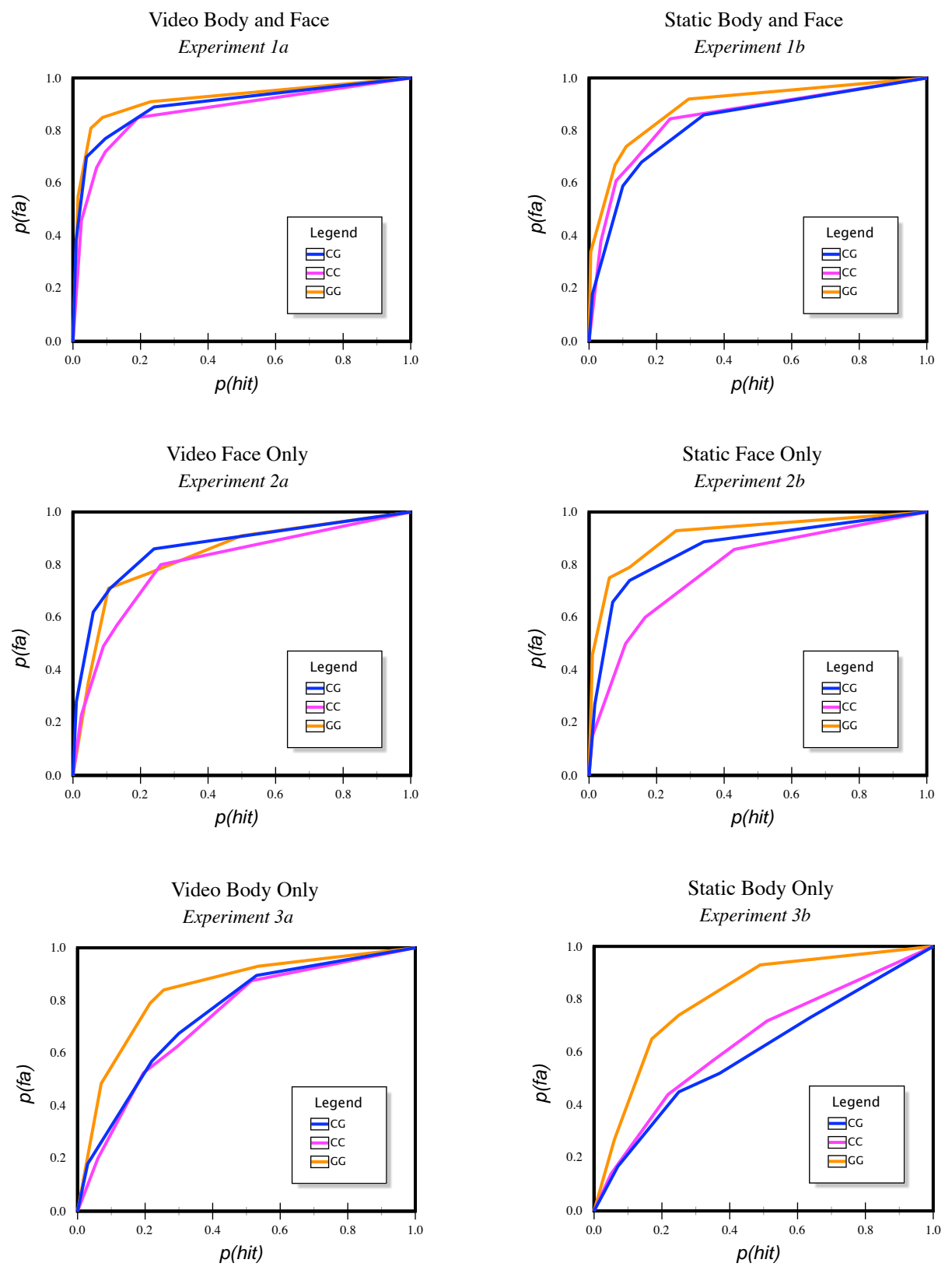

Figure 3: ROC curves for the experiments show a video advantage for the face and body conditions and the body-only condition, but not the face-only condition. They also show a small advantage for the face and body conditions over the face and a stronger advantage for the face-only over body-only condition. There is a reasonably consistent GG advantage over the $\mathrm{CG}$ and $\mathrm{CC}$ conditions. 
nating matched and mismatched identity pairs each individual in each condition of the experiments. This was done by dividing the rating scale into "match judgments" (ratings of 1 or 2, in which the participant said "sure or think" same person) and non-match judgments (ratings of 3, 4 or 5, don't know and sure or think different people). ${ }^{1}$ We tabulated the proportion of hits and false alarms as follows. Hits were defined as match pairs that received ratings of 1 or 2 (i.e., sure or think that they are the same person). False alarms were defined as non-match pairs that received ratings of 1 or 2 .

\subsubsection{Overview Experimental Results}

To examine the effects of video versus static presentation, as well as the kind of information presented (face and body, body, or face), we conducted a two-factor (video/static and information type) meta-anova, combining data across the six experiments. ${ }^{2}$ An overview of the means for these conditions appears in Figure 4. Consistent with the figure, there was a main effect of video versus static presentation, $F(1,199)=17.17, p<.0001$, with video better than static. There was also a main effect of the information presented, $F(2,199)=54.88, p<.0001$, with face and body best, followed by face-only and then body-only. Both main effects were qualified by the presence of a significant interaction between video/static presentation and information type, $F(2,199)=4.81, p<.009$. The source of this interaction can be found in two results involving the face-only conditions. First, static and dynamic presentations were equivalent when only the face was presented. This indicates that observers did not benefit from seeing multiple images of the face from the video, or from the motion of the face in the videos. The lack of a motion effect for the face condition is not surprising as the videos show only rigid rotational and translation movements of the head.

The second component of the interaction is more interesting. This is the equivalence of the static face-only condition and the static face and body condition. It is worth noting that the face images from which the judgments were made in the static condition were included (identically) in the face and body static images. By identically, we mean that the size of the face image in the static face-only presentation was identical to the size of the

\footnotetext{
${ }^{1}$ The placement of this break is somewhat arbitrary, but we verified the results with the second obvious break point between 3 and 4 .

${ }^{2}$ Because the effects of match type (GG, CC, and CG) were relatively consistent across experiments, for simplicity we omitted match type from the meta-anova.
} 


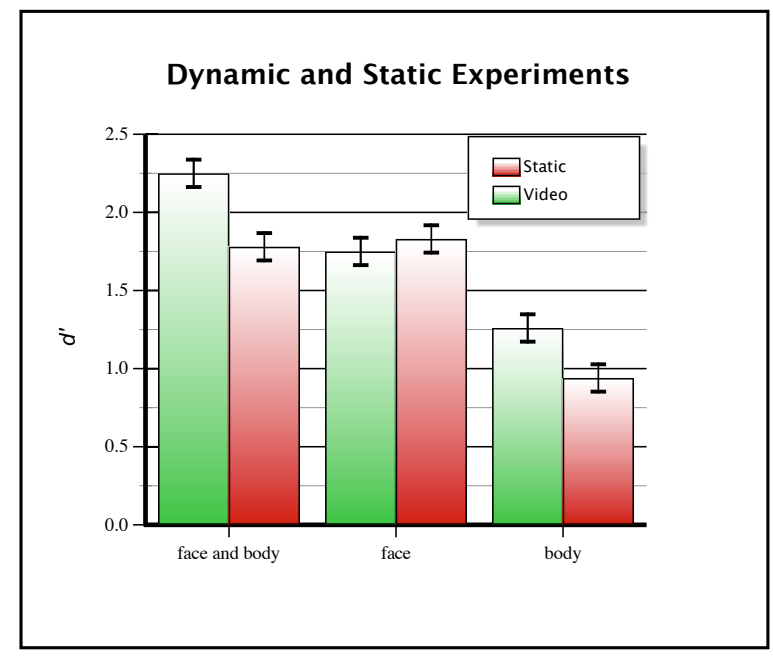

Figure 4: An overview of the means for the first six experiments shows a video advantage for the face and body and body-only conditions. There is also an advantage for the faceonly over the body-only conditions. Of note, the interaction between factors highlights the equivalent performance for a static presentation of the whole person and a static presentation of the face-only.

embedded face in the face and body image. This finding suggests that when observers looked at the full person in a static image, they use only the face for the identity decision. By contrast, in the video presentation conditions, performance was better with the face and body than with the face alone. The fusion data we present shortly offers insight into this interaction.

Combined, the two components of the interaction result in three conditions with roughly equal levels of performance: 1.) static presentation of the face and body; 2.) static presentation of the face only; and 3.) dynamic presentation of the face only. These conditions stand in contrast to a substantial performance advantage for video presentations of the face and body together. Substantially lower performance is seen for the conditions that eliminate the face. These body-only conditions also show a video advantage.

Multi-static controls. Given the video advantage found for the face and body and body-only conditions we conducted a multiple static image version of each of these two conditions. Across the video, static, and multistatic experiments, three patterns of performance are possible. A "pure motion advantage" should yield equivalent performance for the best-static and multi-static conditions. If both the motion and the additional static images 
contribute to the video advantage, performance in the multi-static control should fall between the video and best static condition performance. If the video performance can be accounted for by the multiple images in the video, then the multi-static control condition will be at the same level as the video condition. We found examples of all three patterns in our findings.

The results of the two multi-static control experiments are plotted in Figure 5 along with the video and best static image results. Performance in the multi-static condition, relative to the video and static presentations, yielded no "general" result. Starting with the face and body and body-only presentations, the GG comparison showed a pure video advantage, with the multi-static performance well below the video at the level of the single static presentation. This indicates that the video advantage in the GG conditions comes from using inherently dynamic information for identification. The fact that the pure video advantage appears only in the GG condition, where the motion in both videos (e.g., walking style) is similar enough to be useful for identification, is a further indication of the use of dynamic identity signatures.

At the opposite extreme, in the body-only CG and CC conditions, presentation of multiple static images completely accounted for the video advantage. In the CG face and body condition, both the motion and the extra information in multiple static images contribute to the video advantage. Again, the fusion simulations offer insight into these findings.

Up to this point, the results show that human identification is at its best when the whole person was seen in motion. This indicates that people can benefit from complementary information about the face and body and that seeing the whole person in motion can, in some cases, add to the accuracy of the identification judgment. There was also evidence that performance with the face-only was far better than with the body-only. An interaction between body part and presentation mode suggests that the face "carries" identification in static presentations that include both face and body. Next, we consider the effects of match mode within the experiments.

Within-Experiment Match Mode Comparisons. As noted initially, the primary reason we used different types of match conditions was to diversify the stimulus types, allowing for a more general test of motion versus static presentations and the use of face versus body information. We assumed that differences in this variable would be due to the specifics of the information each provides. To determine the effects of the matching condition (CC,GG, and $\mathrm{CG}$ ), in each experiment the data were submitted to a one-factor analysis of variance (ANOVA) with pair type as a between-subjects factor and $d^{\prime}$ as 

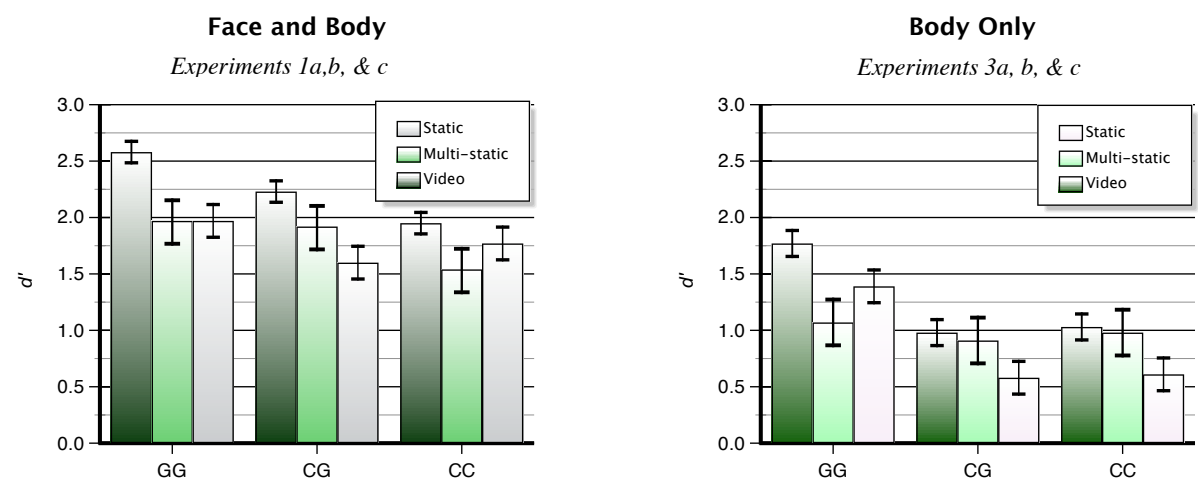

Figure 5: The multi-static control experiments show a range of results from a clear demonstration of the movement in improving performance (GG) to a clear demonstration of multi-static images accounting for the video advantage (CG body-only, CC body-only), to a contribution from both movement and multiple images (CG face and body).

the dependent variable. A summary of results appears in Table 1 and shows statistically different performance across the CC, CG, and GG conditions in all but Exp. 1b, the static face and body match, and in Exp. 3c, the multi-static body match.

As expected, performance with the GG stimuli was generally best. This was true in all but the video face-only experiment. We are uncertain why the video face-only experiment differed from the others for the GG advantage. Across the experiments the ordering of the $\mathrm{CG}$ and $\mathrm{CC}$ conditions varied, but was largely undifferentiated. Of note, for the static presentation of the face there was a relatively strong advantage for the CG multi-modal face comparison over the CC comparison. This seemingly odd result, where matching between images of higher and lower quality is better than matching between two lower quality images, is consistent with previous work (Lui, Seetzen, Burton, and Chaudhuri, 2003). Combined, these results suggests that the higher quality image can bootstrap face processing from the lower quality image.

\section{Fusion}

The purpose of the fusion simulations was to assess more quantitatively how the information presented to participants across the different experi- 
ments can be combined to support more accurate identity judgments. As noted, fusion methods are commonly used in computer vision and biometrics applications when there are multiple, but imperfect, sources of information that are useful for identification. Fusion can improve performance when the contributing information is at least partially independent and when an optimal formula for combining the information generalizes across exemplars. In other words, fusion will improve performance when the information or strategies humans employ in different conditions are complementary. We used fusion here as a tool for assessing how information across these sources is used by humans and to see how the presentation modes (dynamic or static) affect this pattern of use.

It is perhaps worth stressing that even if information is duplicated in conditions (e.g., static face and static face-body), it is nonetheless still possible to improve human performance with fusion. This could occur, for example, if viewing a particular type of stimulus affects the way humans allocate attention to different parts of the stimulus. We will see evidence of this type of effect in the fused combination of conditions.

\section{Methods}

Fusion of the experiments was accomplished with PLS regression, a technique that combines elements of principal component analysis and multiple regression (Abdi, 2003; Naes, Isaksson, Fearn, and Davis, 2004). The technique is used to predict a set of dependent variables from a set of independent variables. The choice of PLS is in part arbitrary and we would expect other pattern classification algorithms to give similar results. We used PLS because it gives a set of easily interpretable weights for individual predictors. PLS yields a set of orthogonal factors, sometimes called latent vectors $t_{1} \ldots t_{l}$ from the covariance matrix of the predictors and dependent variables. The latent vectors are used to predict the dependent variable(s) by appropriately weighting the predictors. The set of weights is referred to as $B_{p l s}$ in the PLS-regression literature.

First, we carried out a fusion that combined identity judgments across

all conditions of the 6 video and static experiments. Based on the results of this first fusion, three additional subset fusions were undertaken, combining data from within the stimulus type conditions (GG, CG and CC) across the body information conditions (face and body, face-only, and body-only). 
Six-experiment fusion. The predictors used in this fusion were the estimates of the match status of the 40 pairs of identities (20 matched identities and 20 mismatched identities) from each of the three conditions (CC, CG, and GG) of the video and static experiments (Exps. 1a and 1b, Exps. 2a and $2 b$, Exps. 3a and 3b). For each pair of images/videos in each condition of each experiment, we averaged the response ratings (i.e., 1 : sure the same person to 5 : sure different people) across participants for the individual identity pairs. ${ }^{3}$ Thus, the predictor for each pair was the average of the participants' ratings of the likelihood that the people were the same. We had 18 such estimates (6 experiments, 3 estimates per experiment) for each pair, that varied based on the type of information (face and body, body, or face) and presentation type (video, static) used in the different experiments. The dependent variable was the actual match status of the pair (same person/different people), quantified as 1 or 0 .

A robust estimate of the fusion performance was determined in a crossvalidation test in which the PLS regression was computed $n$ times with $n-1$ identity pairs and tested with the $n^{\text {th }}$ "left-out" pair. The fusion performance we report is based on the proportion of correct match status classifications of the 40 face pairs. We tested a range of retained PLS factors to find the best performance.

Fusions for $G G, C G$, and $C C$ conditions. Three additional fusions within the stimulus type conditions (GG, CG, and $\mathrm{CC}$ ) were also conducted. For each of these, we extracted the appropriate stimulus type across Experiments 1a, 1b, 2a, 2b, and 3a, 3b. Each of these fusions used 6 predictors (video and static presentations of face and body, face-only and body-only conditions).

\section{Results}

The cross-validation six-experiment fusion classified the match status of the face pairs with $100 \%$ accuracy for both the 3-factor and 4-factor solutions. The weight patterns for these solutions were similar and showed that highvalued weights (i.e., those contributing most strongly) were concentrated in the GG conditions. This is likely due to the general performance advantage

\footnotetext{
${ }^{3}$ To equate the stability of the averages across the different experiments which varied somewhat in number of participants, we averaged the first 10 participants in each experiment.
} 
for the GG conditions across the experiments. For this reason, we divided the fusions into the GG, GC, and CC subset fusions.

The cross-validation fusion for the GG conditions, by itself, yielded perfect match classification accuracy, again for the 3- and 4-factor solutions. Thus, perfect performance was achievable from the information presented in the GG conditions. Again, the pattern of weights for the two solutions were similar, and so we averaged them. These averaged weights appear in Figure 5 and show an intriguing result. The strongly weighted components for the static presentations are from the conditions that include the face (face and body, face-only). For dynamic presentations, the conditions that include the body (face and body, body-only) are strongly weighted. The result suggests that in the static presentation, the face dominates, and the body seems to add little useful information for identification. In the dynamic presentation, however, the body dominates with little independent or complementary contribution from the face. The result also suggests that the combination of the information humans assess most readily from the static presentations (the face) and information assessed most readily from the dynamic presentations (the body) produced perfect identification. Note that the fusion does not indicate how humans combined information across static and dynamic presentations, but rather, how they might combine independent judgments made from the two presentation modes to optimize identification accuracy.

The cross-validation fusion for the CG condition did not achieve perfect match classification, although it did improve classification over the next best condition. The weights in this case, however, were roughly equivalent across all six sub-conditions used in the fusion, suggesting that observers rely on complementary information in each of the six conditions. The CC condition fusion did not improve match status classification accuracy, but rather, in all cross-validation solutions, proved worse than the best input condition. This suggests that there was no formula for combining the identity information across these conditions in a way that generalized across the face pairs. More likely, different combinations of condition-based estimates might be better suited to different subsets of the identity pairs.

\section{Discussion}

When we recognize a person in the real world, we see the whole person, in motion and at rest. In this study, we examined the effects of dynamic and static presentations of the face and body for recognizing people in relatively 


\section{Gait-Gait Fusion}

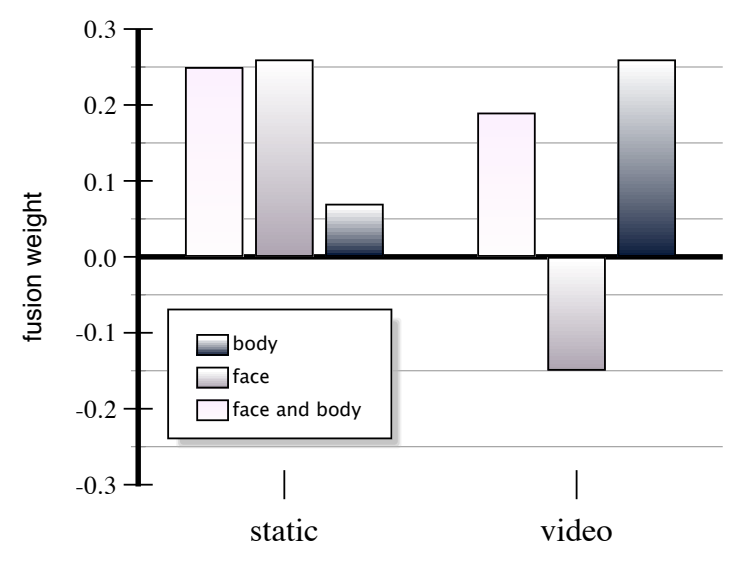

natural viewing conditions. The primary finding of this study is that human identification is at its best when the whole person was seen in motion. This indicates that identification can benefit from both the face and body, and that seeing the whole person in motion can add to the accuracy of the identification judgment. In other words, recognition in the present study was most accurate when the conditions approximated natural viewing conditions.

In dissecting this natural viewing condition advantage, a striking finding was the equivalence of the static face and body and static face-alone conditions. Consistent with previous studies (Burton et al., 1999b; Robbins and Coltheart, 2010), the present data confirm human reliance on the face for identification in static viewing conditions, even when the body is available. From this result, it is tempting to conclude that the static body does not, or cannot, provide useful information for human identification. This conclusion is at odds, however, with the solid performance (i.e., $d^{\prime} \approx 1.0$ ) we found in the static body-alone condition, indicating that humans can use the body for identification. Rather, a better interpretation of the combined findings is that body-based identity information (i.e., structure) is more likely to be used when the face is unavailable, or in real world terms, when viewing conditions for the face are poor.

In neural terms, areas in the inferior temporal cortex, including FFA 
and OFA, are the likely neural sub-strates for face processing from static images. Concomitantly, the use of static body information in the present study accords well with the function proposed for the EBA by Downing et al. (2001). Based on the particular responsiveness of EBA to static bodies, Downing et al. (2001) proposed a role for EBA in representing the visual appearance of bodies when viewing conditions are poor or when the face is not easily accessible due to poor lighting, occlusion, or viewing direction.

The second component of our empirical findings concern the effects of motion on identification. Motion improved identification accuracy when the body was visible. This suggests that the body motions we see in natural viewing conditions can contribute to the visual representation of identity. Of note, these body-based video advantages came from different sources, which we probed by comparing performance in the best-static and dynamic conditions to a multi-static image control condition. The pure motion benefit we found in the gait-to-gait comparisons indicates the use of dynamic identity-signatures for identification and fits with the supplemental information hypothesis (O'Toole et al., 2002), and thus a role for the pSTS in in person recognition. A prerequisite for using this information is that in the gait-to-gait comparisons, there was a match between the types of motion signatures available. Thus, stereotyped walking motions may have provided the supplemental motion-based identity information.

Other body-based video advantages could be accounted for entirely by seeing multiple images of the person. This was clearest for the body-only conversation-conversation and conversation-gait comparisons. This latter is a cross-modal comparison requiring observers to match across rather different image formats. In these cases, we found roughly equal performance for the video and multi-static conditions, at a level that exceeded performance for the best static image condition. Of note, the video/multi-static advantage for the cross-modal case could not have been due to direct image matching processes between the comparison pair. In fact, the images embedded in the conversation and gait videos differed markedly in viewpoint, illumination, distance, and resolution. Rather the match task required observers to compare video/images between a higher quality (gait) and lower quality (conversation) stimulus. Consequently, the video and multi-static advantage had to have been based on active internal processing, whereby multiple images in the sequence are used to create a more robust representation than would be possible with the single image. Previous studies (Lui, Seetzen, Burton, and Chaudhuri, 2003; Roark, Barrett, O'Toole, and Abdi, 2005) have likewise 
shown a kind of bootstrapping from lower to higher quality face recognition. All three findings suggest a process that actively constructs a more robust representation from low-quality stimuli, using internal resources from long term experience with faces and bodies.

It is worth noting that the lack of motion benefit with faces should not be over-interpreted to suggest that we have no representation of facial motion in the identity code. Indeed, previous studies have demonstrated that non-rigid facial motions can be used for identifying someone (Hill and Johnston, 2001; Knappmeyer, Thornton, and Bülthoff, 2003). Under normal conditions, these non-rigid motions are visible only when we view a face from a short distance. At this close distance, movement is generally not needed for identification, because of the high quality of the pictorial codes. Rather, consistent with distributed model, the primary function of non-rigid facial motions is likely to be social.

In the introduction, we proposed that a better understanding of how humans identify people from static and dynamic information in the face and body could constrain the interpretation of the complex neural network of brain areas that respond to faces and bodies. The fusion data offer a functionally based mechanism for applying these constraints to a complex data set. It is worth stressing that the fusion applied here does not tell us specifically how humans used the information in the various conditions, but rather how human identification judgments made in different stimulus and viewing conditions could be combined to optimize accuracy. The fusion results suggest that humans access non-redundant identity information from the face versus body to differing degrees from moving versus static stimuli. Specifically, it indicates that optimal performance can be achieved by combining human observer judgments from static viewing conditions that include the face and dynamic viewing conditions that include the body. One reason for the differential access of face versus body information from moving and static stimuli, may be based on the complex structure of neural areas processing face and body information for different reasons.

In summary, human judgments of identity are likely to be based on the collaborative computations of multiple representations of face and body, and their associated motions in the high-level visual processing network. A knowledge of how humans identify people in natural viewing environments can ground theories of how this identity information interacts in these neural networks. 


\section{References}

Abdi, H., 2003. Partial least squares regression (pls-regression). In: Beck, M. L., Bryman, A., Futing, T. (Eds.), Encyclopedia for Research Methods for the Social Sciences. Thousand Oaks: CA, Sage, pp. 792-795.

Allison, T., Puce, A., McCarthy, G., 2000. Social perception from visual cues: The role of the sts region. Trends in Cognitive Science 4(7), 267-278.

Astafiev, S., Stanely, C., Shuman, G., Corbetta, M., 2004. Iextrastriate body area in human occipital cortex responds to the performance of motor actions. Nature Neuroscience 7.

Burton, A. M., Bruce, V., Hancock, P. J. B., 1999a. From pixels to people: A model of familiar face recognition. Trends in Cognitive Sciences 23, 1-31.

Burton, A. M., Wilson, S., Cowan, M., Bruce, V., 1999b. Face recognition in poor-quality video. Psychological Science 10, 243-248.

Cutting, J. E., Kozlowski, L. T., 1977. Recognizing friends by their walk: gait perception without form cues. Bulletin of the Psychonomic Society 9, 353-356.

Davis, J. P., Valentine, T., 2008. Cctv on trial: Matching video images with the defendant in the dock. Applied Cognitive Psychology 23(4), 482-505.

Downing, P. E., Jiang, Y., Shuman, M., Kanwisher, N., 2001. A cortical area selective for visual processing of the human body. Science 293(5539), $2470-2473$.

Downing, P. E., Peelen, M., Wiggett, A., Tew, B. D., 2006. Is the extrastriate body area involved in motor actions? Nature Neuroscience 1(1), 52-62.

Hancock, P. J. B., Bruce, V., Burton, A. M., 2000. Recognition of unfamiliar faces. Trends in Cognitive Sciences 4, 330-337.

Haxby, J., Hoffman, E., Gobbini, M., 2000. The distributed human neural system for face perception. Trends in Cognitive Science 20(6), 223-233.

Hill, H., Johnston, A., 2001. Categorizing sex and identity from the biological motion of faces. Current Biology 11, 880-885. 
Kanwisher, N., McDermott, J., Chun, M., 1997. The fusiform face area: a module in human extrastriate cortex specialized for face perception. Journal of Neuroscience 17, 4302-4311.

Knappmeyer, B., Thornton, I., Bülthoff, H. H., 2003. The use of facial motion and facial form during the processing of identity. Vision Research 43(18), 1921-1936.

Lui, C. H., Seetzen, H., Burton, A., Chaudhuri, A., 2003. Face recognition is robust with incongruent image resolultion. Journal of Experimental Psychology: Applied 9, 33-44.

Naes, T., Isaksson, T., Fearn, T., Davis, T., 2004. Multivariate calibration and classification. NIR Publications, New York.

O'Toole, A., Roark, D., Abdi, H., 2002. Recognition of moving faces: A psychological and neural perspective. Trends in Cognitive Science 6, 261266.

O'Toole, A. J., Roark, D. A., 2010, in press. Memory for moving faces: The interplay of two recognition systems. In: Dynamic faces: Insights from Experiments and Computation. p. (in press).

Peelen, M., Downing, P., 2005. Is the extrastriate body area involved in motor actions? Nature Neuroscience 8(125), 6996-7001.

Pilz, K. S., Vuong, Q. C., Bülthoff, H. H., Thornton, I. M., 2006. Body motion influences the perception of identity. Perception 35, 210.

Pinsk, M., DeSimone, K., Moore, T., Gross, C., Kastner, S., 2005. Representations of faces and body parts in macaque temporal cortex: A functional mri study. Proceedings of the National Academy of Sciences 102(19), 69967001 .

Roark, D. A., Barrett, S. E., O'Toole, A. J., Abdi, H., 2005. Learning the moves: The effect of facial familiarity and facial motion on person recognition across large changes in viewing format. Perception 35, 761-773.

Robbins, R., Coltheart, M., 2010. Heads, bodies and holistic processing in person recognition. Journal of Vision in press, pp. 
Ross, A., Nandakumar, K., Jain, A., 2004. Handbook of Multibiometrics. Springer-Verlag, New York.

\section{Acknowledgments}

This work was supported by funding from the Technical Support Working Group, U.S. Dept. of Defense, to A. J. O'Toole. 